\title{
The Influence of the Artificial Nanostructure on the LiF Formation at the Solid-electrolyte
} Interphase of Carbon-based Anodes

\author{
Katrine L. Svane, ${ }^{* \dagger}$ Sebastian Zimmer Lefmann,${ }^{\dagger}$ Mads Schousboe Vilmann ${ }^{\dagger}{ }^{\dagger}$ Jan \\ Rossmeisl, ${ }^{\ddagger}$ and Ivano E. Castelli, ${ }^{* \dagger}$ \\ $\dagger$ Department of Energy Conversion and Storage, Technical University of Denmark, Anker \\ Engelundsvej 411, DK-2800 Kgs. Lyngby, Denmark \\ $\ddagger$ Department of Chemistry, University of Copenhagen, Universitetsparken 5, DK-2100 \\ Copenhagen $\varnothing$, Denmark \\ E-mail: kasv@dtu.dk; ivca@dtu.dk
}

\begin{abstract}
The solid-electrolyte interphase (SEI) is of crucial importance for the performance of Li-ion batteries. Here, Density Functional Theory (DFT) calculations are used to study the formation of one of the simplest and early appearing components of the SEI layer, namely LiF, which is produced by splitting HF impurities. The process is investigated on different models representing the basal and edge planes of a graphitic anode, and on covalently connected carbon nanotubes and graphene sheets, known as pillared graphene. The results show that $2 \mathrm{Li}$ atoms are required to bind $\mathrm{F}$ in the final state in order to make the reaction energetically favorable, or alternatively a $\mathrm{H}$ atom must be pre-adsorbed. The Li adsorption energy, and thereby the Li coverage at a given potential, varies for the different carbon structures, demonstrating that the artificial nanostructure of the carbon can influence the formation of the SEI.
\end{abstract}


Li-ion batteries (LiBs) power the majority of portable electronic devices and play an important role in the transition to sustainable energy sources from fossil fuels. Thus, ongoing research is aimed at improving key properties such as storage capacity, power density, safety and durability. ${ }^{1,2}$ Focusing on the anode side,${ }^{3}$ graphite is the dominating material used in LiBs: it shows low cost, good stability and durability, and high Coulombic efficiency, but can only store one Li ion for every six carbons, corresponding to a maximum theoretical capacity of $372 \mathrm{mAh} / \mathrm{g} .{ }^{4}$ Other materials have been investigated as possible replacements for graphite: Li-metal anodes have a much higher energy density, but they also suffer from the formation of dendrites that short circuit the battery; ${ }^{5}$ silicon has 10 times the storage capacity of graphite, but also exhibit a 300\% volume expansion during cycling, which makes the interface unstable. ${ }^{6}$ Artificial nano-engineered and composite materials are possible solutions to increase the capacity of graphite and solve the issues with volume. ${ }^{7-10}$ One example of an artificially nanostructured carbon material is the so-called pillared graphene, which consists of covalently connected graphene and carbon nanotubes (CNTs). This material was first theoretically designed for hydrogen storage, ${ }^{11}$ but experimental realizations of graphene-CNT connected materials have resulted in improvements of several other technologies including supercapacitors, solar cells and anodes with increased storage capacity in LIBs. ${ }^{12-15}$

The choice of anode material not only affects the capacity of the battery, but also determines the formation of the solid-electrolyte interphase (SEI) which ultimately influences the performance and safety of a LIB. The SEI layer is formed by decomposition of the electrolyte during the initial potential cycles of the battery and serves to protect the electrode from electrons and thereby further reduction, while allowing the passage of $\mathrm{Li}^{+}$ions during intercalation and deintercalation. However, in spite of numerous experimental and theoretical research efforts, many aspects of the SEI formation are still not fully understood. ${ }^{16-18}$ The development of artificial nanostructured materials and interfaces can, in addition to improving bulk level properties such as the storage capacity, potentially solve some of the issues encountered for conventional anode electrode interfaces. The lack of edges in pillared 
graphene compared with the edge planes of graphite, for example, might change the reduction reactions of the electrolyte and impurities. Despite this potential impact in many applications, the role of the electrochemical interface in nano-architectured materials is often not investigated and is considered subsequential to the optimization of the bulk properties of the anode. ${ }^{19}$ Being able to simultaneously improve the properties of the bulk electrode and proactively control the SEI layer by nanostructuring the interface is one of the "great challenges" of the battery community.

One of the simplest components of the SEI, and one of the first to be formed during the initial cycles of a battery, is $\mathrm{LiF} .{ }^{1}$ Recent studies have shown that the LiF formation can happen when $\mathrm{Li}$ is pre-adsorbed on the interface by the electrochemical decomposition of HF impurities. ${ }^{20,21}$ It was highlighted that the overpotential necessary to run the reduction reaction is related to the formation of the correct interface, i.e. the adsorption of Li, rather than providing the reaction energy. Moreover, the potential at which Li starts to be present at the interface was found to be correlated with both the work function of the electrode and the Li adsorption energy. In this paper, we use Density Functional Theory (DFT) calculations to study the formation of LiF from HF on various models representative of carbon-based anode materials. Our results show that for all the investigated models the splitting of HF is only energetically favorable if $\mathrm{H}$ is already chemisorbed on the carbon or, alternatively, if the coverage of $\mathrm{Li}$ is sufficiently high that two Li atoms can stabilize the final state. The Li adsorption energy, and thereby the coverage of Li at a given potential varies for the different carbon models, suggesting that the carbon nanostructure affects not only the storage capacity but also the interface properties.

Firstly, two carbon-based models are considered, representing the basal plane of the commonly used graphite anode: defect-free graphene (Figure 1a) and graphene with a StoneWales (SW) defect ${ }^{22}$ (Figure 1b). In addition three different models of pillared graphene are investigated, one of which is shown in Figure 1c-e (c.f. Section S1 of the ESI for images of the two others). The three models differ in the way the carbon nanotube is joined with the 

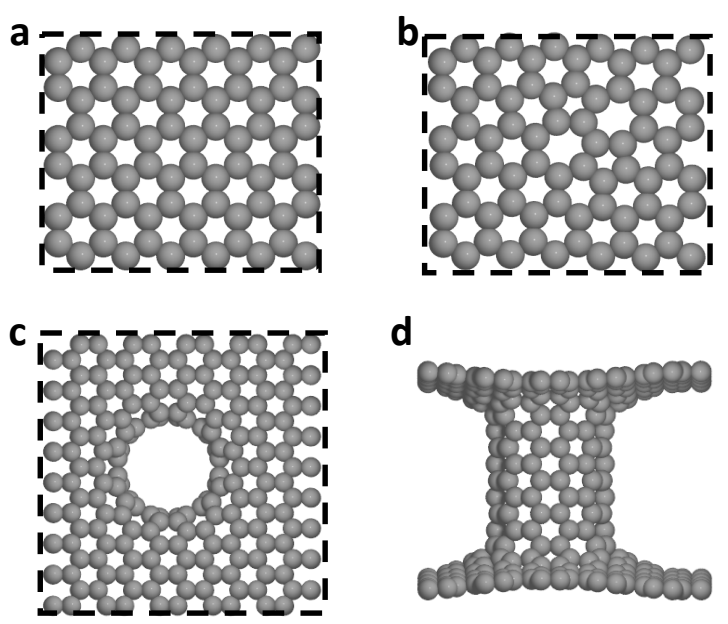

d
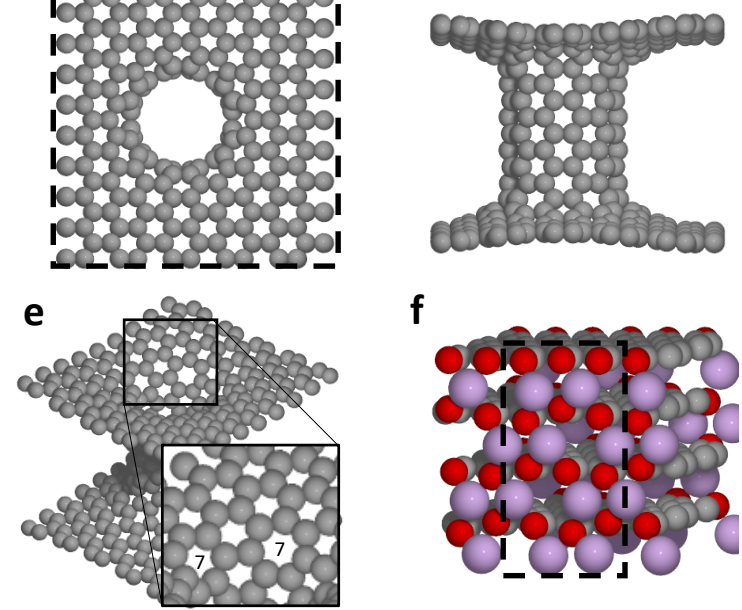

Figure 1: Carbon-based anode models considered in this paper: a) graphene, b) graphene with a Stone-Wales defect, c) pillared graphene with the $\mathbf{6 h e p t}$ connection between the carbon nanotube and the graphene sheet in top and d) side view and e) tilted with an inset showing the heptagons at the connection and f) O-terminated graphite edge plane with 2/3 $\mathrm{Li}$ coverage. Carbon is grey, $\mathrm{O}$ is red and $\mathrm{Li}$ is purple. The computational unit cell is indicated with black dashed lines in $\mathrm{a}, \mathrm{b}, \mathrm{c}$ and $\mathrm{f}$. 
graphene sheet, which results in 3 octagons, 6 heptagons (shown in Figure 1c-e) or 6 octagons and 6 pentagons being present at the seam, and they are therefore named 3oct, 6 hept and 6oct +6 pent in the following. ${ }^{11}$ The calculated formation energy relative to carbon in graphene follows the order $\mathbf{6 h e p t}<\mathbf{3 o c t}<6$ oct $+\mathbf{6 p e n t}$ (c.f. Section S1 in the ESI). Since the synthesis procedure could favor one of the energetically less favorable structures or lead to a mixture of these connection types all three models have been investigated. After discussing the results obtained on these carbon-only models, they are compared with results obtained on a model of an oxygen-terminated graphite edge. The edge-plane is considered to be the facet at which Li intercalation and deintercalation happens, and therefore presents a highly relevant model for the real graphitic anode. The detailed structure of the graphite edge plane in batteries at operating conditions is difficult to determine and may vary depending on the preparation, but oxidation of the surface has been found to improve performance and reduce graphite exfoliation and the dominance of zigzag or armchair edges have a direct effect on the formation of the SEI layer. ${ }^{23-27}$ The edge-plane model used here is therefore an oxygen-terminated zigzag edge. The purely O-terminated edge adsorbs Li very strongly, with a calculated adsorption energy of $-3.84 \mathrm{eV}$ relative to Li metal at a coverage of $1 / 12$, and some $\mathrm{Li}$ is therefore expected to be adsorbed even without the application of an overpotential. This could affect the reduction reaction of $\mathrm{HF}$ and other impurities, as an overpotential may then only be required to overcome any reaction barriers, instead of providing the energy to build an interface with a sufficient coverage of Li, as pointed out for single metal surfaces. ${ }^{21}$ Previous studies of O-terminated graphite showed a strong average adsorption energy of Li even at full coverage, ${ }^{28}$ but the differential adsorption energy decreases with coverage. The results given here are for a model with a Li coverage of $2 / 3$ (Figure 1f), at which point the differential adsorption energy of $\mathrm{Li}$ is $-1.18 \mathrm{eV}$. (c.f. Section S1 ESI for further details of this model and results for selected alternative coverages of $\mathrm{Li}$ ).

The structures are optimized using DFT as further detailed in the Computational Methods section. Firstly, the adsorption of Li is investigated, as it can be correlated with the 
Li-adsorption potential. ${ }^{21}$ For the carbon-only models, $\mathrm{Li}$ adsorbs $\mathrm{as}^{+}$at the center of carbon rings, resulting in only one inequivalent site on the graphene model, three on graphene with a SW defect and a large number of sites for the pillared graphene models, spanning positions on the inside and the outside of the tube, at the edge of the tube and on the flat part of the structure. The preferred adsorption site and the corresponding adsorption energy is given in Table 1 for the various graphene models and further details of other sites can be found in Section S3 of the ESI. In agreement with previous calculations, the adsorption of $\mathrm{Li}$ relative to $\mathrm{Li}(\mathrm{s})$ is not calculated to be favorable on defect-free graphene while it is close to zero for the SW defect. ${ }^{29}$ On pillared graphene, the energy varies with position, for all three models being lowest at the defects in the convex region connecting the graphite sheet and the inside of the carbon nanotube. The disruptions to the perfect $s p^{2}$ bonding of graphene induced by convex curvature and defects have previously been shown to make the covalent bonding of hydrogen (chemisorption) more favorable. ${ }^{30,31}$ Focus will therefore be on these regions in the following investigations of the HF splitting, since the $\mathrm{H}$ is assumed to chemisorb on the surface before possibly recombining with another $\mathrm{H}$ to form $\mathrm{H}_{2}(\mathrm{~g}) .{ }^{21}$

Table 1: Li adsorption energies $\left(\mathrm{E}_{\mathrm{ads}}\right)$ on the various carbon models relative to $\mathrm{Li}(\mathrm{s})$. All values are in $\mathrm{eV}$.

\begin{tabular}{lll}
\hline Model & Site & $\mathrm{E}_{\text {ads }}$ \\
\hline Graphene & 6-ring & 0.38 \\
Stone-Wales & 7-ring & 0.05 \\
3oct & 8-ring, convex edge & -0.27 \\
6hept & 7-ring, convex edge & -0.12 \\
6oct + 6pent & 5-ring, convex edge & -0.79 \\
& 8-ring, convex edge & -0.79 \\
\hline
\end{tabular}

The most favorable $\mathrm{H}$ chemisorption positions are identified on the various carbon models (c.f. Section S4 in the ESI for details). It is well known that the chemisorption energy of $\mathrm{H}$ on graphene depends on the number of hydrogens already present, and the chemisorption energies for a second $\mathrm{H}$, assumed to adsorb on one of the neighboring sites following previous reports, is therefore also calculated. ${ }^{32}$ The resulting preferred chemisorption site of $1 \mathrm{H}$ and chemisorption energies of the first and second $\mathrm{H}$ relative to $\mathrm{H}_{2}(\mathrm{~g})$ are given in Table 2. The 
results show that the chemisorption of one $\mathrm{H}$ atom on graphene is very unfavorable, and in fact, if $\mathrm{Li}$ is present $\mathrm{H}$ prefers to adsorb on $\mathrm{Li}$ as $\mathrm{H}^{-}$with an adsorption energy of $0.80 \mathrm{eV}$. The planar defects in the SW model lower the chemisorption energy considerably, as do the curved defect regions at the edge of the pillared graphene models. In the case of the $\mathbf{6 o c t}+\mathbf{6}$ pent structure, the chemisorption of $\mathrm{H}$ is found to be thermoneutral on the site between a 5-ring and an 8-ring. This region also has the strongest adsorption of Li, indicating that the defect configuration is relatively unstable. The chemisorption of a second $\mathrm{H}$ requires significantly less energy on all of the considered surfaces, and on the 6oct +6 pent and 6 hept structures the total enthalpy for splitting of $\mathrm{H}_{2}$ is negative by $-0.78 \mathrm{eV}$ and $-0.27 \mathrm{eV}$, respectively. Depending on the free energy contributions to the energy under relevant conditions it is therefore possible that some of these sites will be occupied by $\mathrm{H}$ rather than evolving $\mathrm{H}_{2}(\mathrm{~g})$.

Table 2: Most favorable $\mathrm{H}$ chemisorption site and chemisorption energies $\left(\mathrm{E}_{\mathrm{chem}}\right)$ of the first and second hydrogens on the various carbon models. The chemisorption site is specified by listing the neighboring carbon rings that are not 6-rings. All energies are in $\mathrm{eV}$.

\begin{tabular}{llll}
\hline Model & Site & $\begin{array}{l}\mathrm{E}_{\text {chem }} \\
(1 \mathrm{st} \mathrm{H})\end{array}$ & $\begin{array}{l}\mathrm{E}_{\text {chem }} \\
(2 \mathrm{nd} \mathrm{H})\end{array}$ \\
\hline Graphene & 6-ring & 1.55 & 0.37 \\
Stone-Wales & 5-ring $+2 \times 7$-ring & 0.59 & -0.11 \\
3oct & 8-ring, edge & 0.34 & 0.03 \\
6hept & 7-ring, edge & 0.55 & -0.82 \\
6oct + 6pent & 5-ring + 8-ring, edge & -0.03 & -0.75 \\
\hline
\end{tabular}

The mechanism for HF splitting and LiF formation is now considered. The splitting of $\mathrm{HF}$ in the absence of Li proceeds from an initial state with HF physisorbed on the surface (Figure 2a). In the final state it is assumed that either $\mathrm{H}$ or $\mathrm{F}$ occupies the most favorable chemisorption position found for $1 \mathrm{H}$ above, and the other atom is chemisorbed on a neighboring carbon atom (Figure $2 \mathrm{~b}$ ). Calculating the energies of these possible configurations reveals that $\mathrm{H}$ and $\mathrm{F}$ preferably chemisorb on the same two sites as $2 \mathrm{H}$ on all of the models (c.f. ESI for images of these sites for all models). Figure 2c shows the energy diagram for the process, clearly revealing that splitting of $\mathrm{HF}$ in the absence of $\mathrm{Li}$ is unfavorable on all the 
structures. A relatively small energy of $0.34 \mathrm{eV}$ is required on the 6 oct $+\mathbf{6 p e n t}$ structure, again suggesting that the defects in this structure are more reactive than those in any of the other structures.

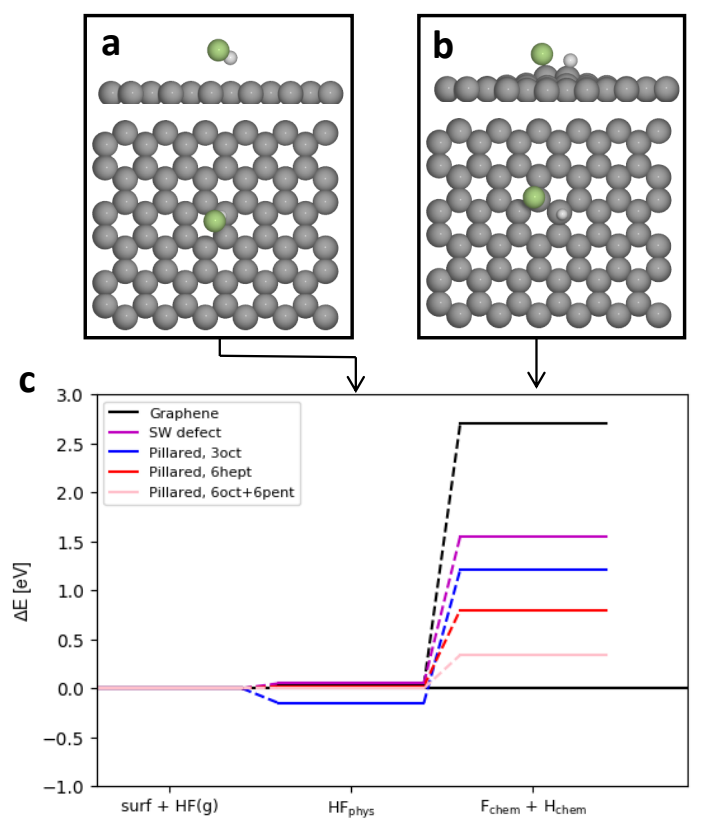

Figure 2: Structures of the relevant intermediates for splitting of HF on graphene in the absence of $\mathrm{Li}$ : a) physisorbed $\mathrm{HF}$ on the surface and b) chemisorbed $\mathrm{H}+\mathrm{F}$. c) Corresponding energy diagram for the process on the different carbon models. Carbon is grey, $\mathrm{F}$ is green and $\mathrm{H}$ is white.

In the presence of one adsorbed $\mathrm{Li}$ atom $\left({ }^{*} \mathrm{Li}\right)$ the splitting of $\mathrm{HF}$ is assumed to follow the mechanism discussed in Ref. 20 and 21, which is illustrated in Figure 3a-c:

$$
\begin{aligned}
& { }^{*} \mathrm{Li}+\mathrm{HF}_{\text {phys }} \longrightarrow{ }^{*} \mathrm{LiFH} \\
& { }^{*} \mathrm{LiFH} \longrightarrow{ }^{*} \mathrm{LiF}+\mathrm{H}_{\text {chem }}
\end{aligned}
$$

Initially the Li atom is assumed to adsorb in the most favorable position on the surface and $\mathrm{HF}$ is physisorbed some distance away $\left({ }^{*} \mathrm{Li}+\mathrm{HF}_{\text {phys }}\right.$, Figure $\left.3 \mathrm{~b}\right)$. HF then adsorbs in its undissociated form on the $\mathrm{Li}$ atom $\left({ }^{*} \mathrm{LiFH}\right.$, Figure $\left.3 \mathrm{c}\right)$, and is subsequently split into $\mathrm{H}$ chemisorbed in its most favorable position on the surface and $\mathrm{F}$ remaining on the $\mathrm{Li}$ 
atom $\left({ }^{*} \mathrm{LiF}+\mathrm{H}_{\text {chem }}\right.$, Figure $\left.3 \mathrm{~d}\right)$. The corresponding energy diagram is shown in Figure 3a for the various models. The results reveal that it is favorable for HF to adsorb on Li on all of the surfaces, but the subsequent splitting is endothermic, partly because the final state involves a chemisorbed $\mathrm{H}$ atom. Since the chemisorption of a second $\mathrm{H}$ atom is more favorable than that of the first (Table 2) the last step is expected to be more favorable if the same reaction proceeds with a spectator $\mathrm{H}$ chemisorbed on the surface. The energy diagram for this situation is shown in Figure 3e, and indeed the splitting of ${ }^{*} \mathrm{LiFH}$ to ${ }^{*} \mathrm{LiF}$ and $\mathrm{H}_{\text {chem }}$ becomes thermoneutral or exothermic on all of the considered models. Of course, an overpotential is still needed to adsorb Li on the surface, and this potential would most likely be larger than the one required for the chemisorption of $\mathrm{H}$. The presence of $\mathrm{Li}$ is, in fact, required to split HF, also when $\mathrm{H}$ is chemisorbed, as it has been shown for single metal surfaces. $^{21}$

The splitting of HF was also considered for the case where two Li atoms are present on the surface, since $2 \mathrm{Li}$ was found to be necessary in order to split $\mathrm{H}_{2} \mathrm{O}$ on $\mathrm{Au}$ and $\mathrm{Pt} .{ }^{21}$ The energy diagram in Figure 4a is calculated assuming that HF initially adsorbs on one of the $\mathrm{Li}$ atoms and the second $\mathrm{Li}$ is adsorbed some distance away, $\left({ }^{*} \mathrm{LiFH}+{ }^{*} \mathrm{Li}\right.$, Figure $\left.4 \mathrm{c}\right)$ since in some cases structures containing HF adsorbed between two Li atoms spontaneously split $\mathrm{HF}$ during relaxation. In the final structure the Li atom has moved closer such that $\mathrm{F}$ is bound between the two $\mathrm{Li}$ atoms and the $\mathrm{H}$ is chemisorbed on the most favorable adsorption site on the respective models $\left({ }^{*} \mathrm{LiFLi}^{*}+\mathrm{H}_{\mathrm{chem}}\right.$, Figure $\left.4 \mathrm{~d}\right)$. The energy diagram in Figure $4 \mathrm{a}$ clearly shows that the presence of $2 \mathrm{Li}$ atoms makes the splitting of HF favorable. Again the formation of the initial state, in particular the adsorption of $2 \mathrm{Li}$ atoms, might pose a barrier to the reaction. The coverage of Li atoms on the substrate depends on the adsorption energy, which is related to the work function of the material, and increases when the potential is lowered on the $\mathrm{Li} / \mathrm{Li}^{+}$scale. ${ }^{21}$ Thus, an overpotential is required to create the right interface for the reaction to proceed. According to the Li adsorption energies in Table 1 the adsorption of $\mathrm{Li}$ is more favorable on the pillared graphene structures, and the formation of LiF on these 

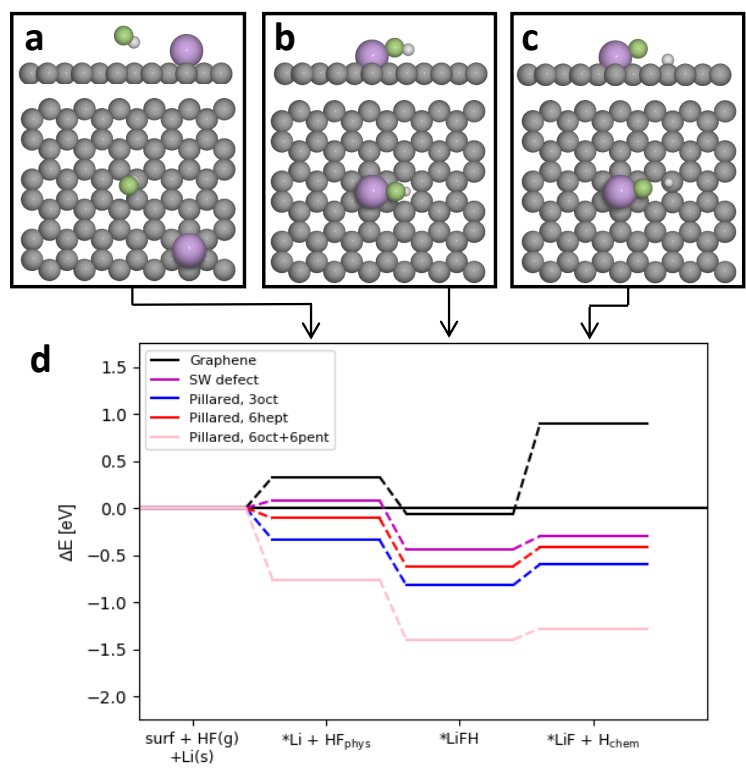

e

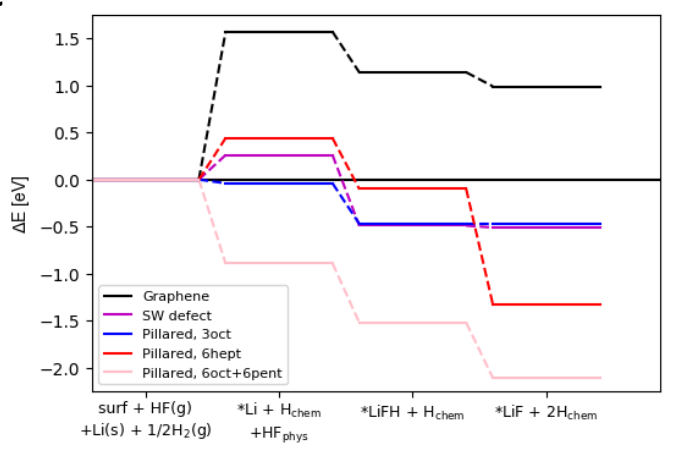

Figure 3: Structures of the relevant intermediates for splitting of $\mathrm{HF}$ in the presence of 1Li on graphene: a) separated adsorbed Li atom and physisorbed HF on the surface, b) HF adsorbed on $\mathrm{Li}$ and c) $\mathrm{F}$ adsorbed on $\mathrm{Li}$ and $\mathrm{H}$ chemisorbed on the surface. d) Corresponding energy diagram for the process on the different carbon models and e) energy diagram for the same reaction but in the case where $1 \mathrm{H}$ is adsorbed on the surface before the splitting of HF. Carbon is grey, Li is purple, $\mathrm{F}$ is green and $\mathrm{H}$ is white. 
surfaces is therefore expected to happen at a lower overpotential than on the basal plane of graphite.

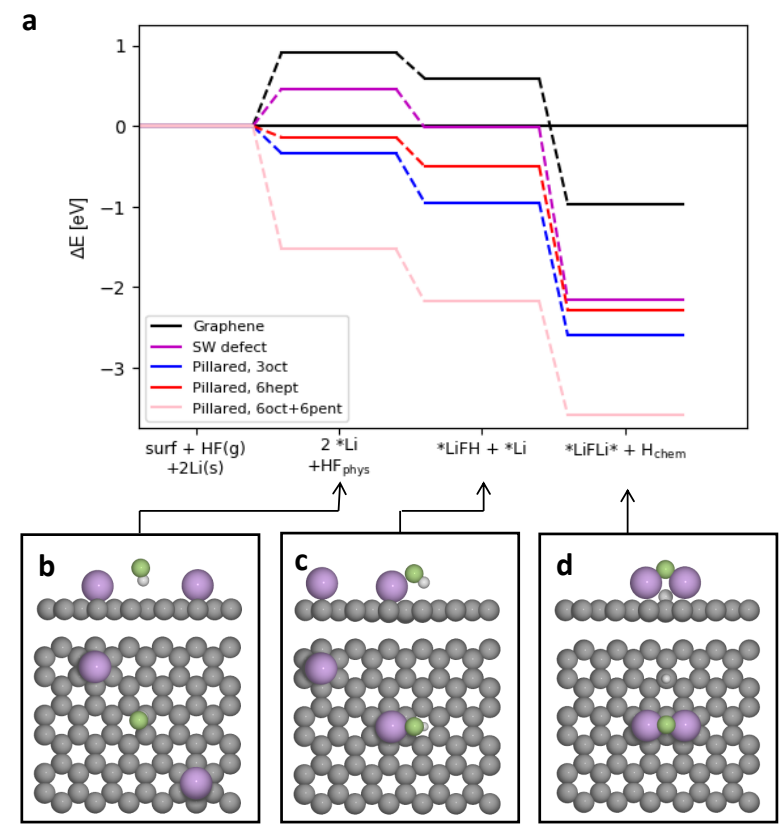

Figure 4: a) Energy diagram for splitting of HF in the presence of 2Li atoms on the different carbon models and top and side views of the relevant intermediates on graphene: b) 2 separated $\mathrm{Li}$ and HF adsorbed on the surface, c) HF adsorbed on Li and one separate $\mathrm{Li}$ atom and d) F adsorbed between two Li atoms and $\mathrm{H}$ chemisorbed on the surface. Carbon is grey, $\mathrm{Li}$ is purple, $\mathrm{F}$ is green and $\mathrm{H}$ is white.

For comparison with a more realistic model of the surface relevant for intercalation in graphite-based anodes, the splitting of HF is now considered on our model of the partly Licovered O-terminated edge plane of graphite (Figure 1f). The reaction energy is calculated starting from the situation where HF adsorbs on a $\mathrm{Li}$ atom next to a Li vacancy such that a strong H-bond can be formed with one of the O atoms of the graphite edge (c.f. figure S3 in the ESI). The $\mathrm{H}$ then jumps to the oxygen and the $\mathrm{F}$ atom moves to a position between two Li atoms, lowering the energy by $1.10 \mathrm{eV}$. The reaction energy depends on the coverage of $\mathrm{Li}$, but it is negative for investigated coverages between $7 / 12$ and $10 / 12$. Only at very low coverages, i.e. when $\mathrm{F}$ does not bind with $2 \mathrm{Li}$ in the final configuration, is the reaction found to be unfavorable (c.f. Section S1 of the ESI for further details). Because a high 
coverage of $\mathrm{Li}$ is reached at a lower overpotential than on any of the graphene and pillared graphene models, the formation of $\mathrm{LiF}$ is expected to be easier on the O-terminated edge of graphite. These results compare well with experimental studies of graphite, showing the SEI on the edge-plane to be thicker and contain more LiF than that on the basal plane. ${ }^{33,34}$

In summary, we have investigated the formation of LiF by splitting of HF impurities on various models of graphite and pillared graphene. The results show that the process is more favorable if $\mathrm{F}$ binds to $2 \mathrm{Li}$ atoms in the final state or, for the graphene and pillared graphene models, if $1 \mathrm{H}$ is already chemisorbed on the surface. For these models both the ionic interaction of $\mathrm{Li}$ and the chemisorption of $\mathrm{H}$ is found to be stronger on the defective convex regions of pillared graphene, and $\mathrm{LiF}$ is therefore expected to form here at a lower overpotential than on perfect graphene. The O-terminated edge of graphite adsorbs Li very strongly, and is therefore expected to have the lowest overpotential for HF splitting. Since pillared graphene (in principle) is free of any edges, LiF formation on an electrode made of this material would happen at a higher overpotential than on a graphite electrode. The importance of LiF in the SEI is not fully understood, and therefore the extent to which this is an advantage is unknown. Furthermore, we have only considered the initial nucleation process and not how LiF grows or how it is affected by competing processes. Nevertheless, our results demonstrate that the (artificial) nanostructure of the carbon in the battery anode affects the formation of one of the main SEI components, in addition to affecting the capacity, both of which are highly important for the performance of the battery.

\section{Computational Methods}

Density functional theory calculations were performed using the Grid-based Projector Augmented Wave (GPAW) ${ }^{35,36}$ software, and the Atomic Simulation Environment (ASE) ${ }^{37}$ was used for setting up and visualizing the structures. The exchange and correlation energy was described using the RPBE functional. ${ }^{38}$ Due to the large size of the pillared graphene models, structural optimization is performed in the Linear Combination of Atomic Orbitals 
(LCAO) mode ${ }^{39}$ until the forces on all atoms are below $0.05 \mathrm{eV}^{-1}$ and the final energy of the optimized structure is evaluated on a real-space grid with a grid-spacing of $0.2 \AA$. This eliminates the effect of basis set superposition errors which can otherwise be a problem for calculations performed in LCAO mode. For details on the accuracy of this procedure see the ESI. Periodic boundary conditions are used in the $x$ - and $y$-directions while non-periodic boundary conditions with a minimum of $6.5 \AA$ between the atoms and the edge of the cell is used in the $z$-direction. The large unit cells for the pillared graphene (ca. $21 \times 22 \AA$ ) are sampled with a single k-point at $\Gamma$ while the models of a single graphene sheet (with or without a SW defect), which have a size of $14.9 \times 12.9 \AA$ are optimized with $3 \times 3 \mathrm{k}$-points and the final energy is calculated with $5 \times 5 \mathrm{k}$-points. The lattice constant of graphene is optimized in the $x y$-plane $(a=b=2.485 \AA)$, but since vdW-interactions are not well described by the RPBE functional the model of the graphite edge plane is set up with the experimentally determined lattice parameter of $6.71 \AA$ along the $z$-direction. ${ }^{40} \mathrm{~A} 3 \mathrm{x} 3$ model with 4 layers and a zigzag edge terminated by $\mathrm{O}$ atoms is used to model the graphite edge plane, and this structure is sampled by $5 \times 2 \mathrm{k}$-points. Further details of the structural models can be found in section S1 of the ESI.

\section{Acknowledgement}

The authors would like to thank Prof. G. Froudakis for sharing the pillared graphene structures used in this work.

\section{Supporting Information Available}

Supporting information available: PDF with further computational details. DFT optimized structures are available from DOI:10.5281/zenodo.3975735 [Link activated upon acceptance]. 


\section{References}

(1) Etacheri, V.; Marom, R.; Elazari, R.; Salitra, G.; Aurbach, D. Challenges in the development of advanced Li-ion batteries: a review. Energy Environ. Sci. 2011, 4, 3243-3262.

(2) Goodenough, J. B.; Park, K.-S. The Li-Ion Rechargeable Battery: A Perspective. Journal of the American Chemical Society 2013, 135, 1167-1176.

(3) Andre, D.; Hain, H.; Lamp, P.; Maglia, F.; Stiaszny, B. Future high-energy density anode materials from an automotive application perspective. Journal of Materials Chemistry A 2017, 5, 17174-17198.

(4) Passerini, S.; Scrosati, B. Lithium and Lithium-Ion Batteries: Challenges and Prospects. Interface magazine 2016, 25, 85-87.

(5) Rong, G.; Zhang, X.; Zhao, W.; Qiu, Y.; Liu, M.; Ye, F.; Xu, Y.; Chen, J.; Hou, Y.; Li, W.; Duan, W.; Zhang, . Y. Liquid-Phase Electrochemical Scanning Electron Microscopy for In Situ Investigation of Lithium Dendrite Growth and Dissolution. Advanced Materials 2017, 29, 1606187.

(6) Jin, Y.; Zhu, B.; Lu, Z.; Liu, N.; Zhu, J. Challenges and Recent Progress in the Development of Si Anodes for Lithium-Ion Battery. Advanced Energy Materials 2017, 7, 1700715.

(7) Qi, W.; Shapter, J. G.; Wu, Q.; Yin, T.; Gao, G.; Cui, D. Nanostructured anode materials for lithium-ion batteries: principle, recent progress and future perspectives. J. Mater. Chem. A 2017, 5, 19521-19540.

(8) Cai, C.; Wang, Y. Novel Nanocomposite Materials for Advanced Li-Ion Rechargeable Batteries. Materials 2009, 2, 1205-1238.

(9) Rahman, M. A.; Song, G.; Bhatt, A. I.; Wong, Y. C.; Wen, C. Nanostructured Silicon 
Anodes for High-Performance Lithium-Ion Batteries. Advanced Functional Materials 2015, 26, 647-678.

(10) Xiong, Z.; Yun, Y.; Jin, H.-J. Applications of Carbon Nanotubes for Lithium Ion Battery Anodes. Materials 2013, 6, 1138-1158.

(11) Dimitrakakis, G. K.; Tylianakis, E.; Froudakis, G. E. Pillared Graphene: A New 3-D Network Nanostructure for Enhanced Hydrogen Storage. Nano Letters 2008, 8, 31663170.

(12) Paul, R. K.; Ghazinejad, M.; Penchev, M.; Lin, J.; Ozkan, M.; Ozkan, C. S. Synthesis of a Pillared Graphene Nanostructure: A Counterpart of Three-Dimensional Carbon Architectures. Small 2010, 6, 2309-2313.

(13) Du, F.; Yu, D.; Dai, L.; Ganguli, S.; Varshney, V.; Roy, A. K. Preparation of Tunable 3D Pillared Carbon Nanotube-Graphene Networks for High-Performance Capacitance. Chemistry of Materials 2011, 23, 4810-4816.

(14) Xue, Y.; Ding, Y.; Niu, J.; Xia, Z.; Roy, A.; Chen, H.; Qu, J.; Wang, Z. L.; Dai, L. Rationally designed graphene-nanotube 3D architectures with a seamless nodal junction for efficient energy conversion and storage. Science Advances 2015, 1, 1400198.

(15) Wang, W.; Ruiz, I.; Guo, S.; Favors, Z.; Bay, H. H.; Ozkan, M.; Ozkan, C. S. Hybrid carbon nanotube and graphene nanostructures for lithium ion battery anodes. Nano Energy 2014, 3, $113-118$.

(16) Wang, A.; Kadam, S.; Li, H.; Shi, S.; Qi, Y. Review on modeling of the anode solid electrolyte interphase (SEI) for lithium-ion batteries. npj Computational Materials 2018, 4,15 .

(17) Peled, E.; Menkin, S. Review-SEI: Past, Present and Future. Journal of The Electrochemical Society 2017, 164, A1703-A1719. 
(18) An, S. J.; Li, J.; Daniel, C.; Mohanty, D.; Nagpure, S.; Wood, D. L. The state of understanding of the lithium-ion-battery graphite solid electrolyte interphase (SEI) and its relationship to formation cycling. Carbon 2016, 105, $52-76$.

(19) Liang, Y.; Zhang, W.; Wu, D.; Ni, Q.-Q.; Zhang, M. Q. Interface Engineering of CarbonBased Nanocomposites for Advanced Electrochemical Energy Storage. Advanced Materials Interfaces 2018, 5, 1800430.

(20) Strmcnik, D.; Castelli, I. E.; Connell, J. G.; Haering, D.; Zorko, M.; Martins, P.; Lopes, P. P.; Genorio, B.; Østergaard, T.; Gasteiger, H. A.; Maglia, F.; Antonopoulos, B. K.; Stamenkovic, V. R.; Rossmeisl, J.; Markovic, N. M. Electrocatalytic transformation of $\mathrm{HF}$ impurity to $\mathrm{H} 2$ and $\mathrm{LiF}$ in lithium-ion batteries. Nature Catalysis 2018, $1,255-262$.

(21) Castelli, I. E.; Zorko, M.; Østergaard, T. M.; Martins, P. F. B. D.; Lopes, P. P.; Antonopoulos, B. K.; Maglia, F.; Markovic, N. M.; Strmcnik, D.; Rossmeisl, J. The role of an interface in stabilizing reaction intermediates for hydrogen evolution in aprotic electrolytes. Chem. Sci. 2020, 11, 3914-3922.

(22) Stone, A.; Wales, D. Theoretical studies of icosahedral C60 and some related species. Chemical Physics Letters 1986, 128, $501-503$.

(23) Peled, E.; Menachem, C.; Bar-Tow, D.; Melman, A. Improved Graphite Anode for Lithium-Ion Batteries Chemically: Bonded Solid Electrolyte Interface and Nanochannel Formation. Journal of The Electrochemical Society 1996, 143, L4-L7.

(24) Spahr, M. E.; Wilhelm, H.; Joho, F.; Panitz, J.-C.; Wambach, J.; Novák, P.; DupontPavlovsky, N. Purely Hexagonal Graphite and the Influence of Surface Modifications on Its Electrochemical Lithium Insertion Properties. Journal of The Electrochemical Society 2002, 149, A960. 
(25) Spahr, M. E.; Wilhelm, H.; Palladino, T.; Dupont-Pavlovsky, N.; Goers, D.; Joho, F.; Novák, P. The role of graphite surface group chemistry on graphite exfoliation during electrochemical lithium insertion. Journal of Power Sources 2003, 119-121, 543 - 549, Selected papers presented at the 11th International Meeting on Lithium Batteries.

(26) Ng, S.; Vix-Guterl, C.; Bernardo, P.; Tran, N.; Ufheil, J.; Buqa, H.; Dentzer, J.; Gadiou, R.; Spahr, M.; Goers, D.; Novák, P. Correlations between surface properties of graphite and the first cycle specific charge loss in lithium-ion batteries. Carbon 2009, $47,705-712$.

(27) Bernardo, P.; Meins, J.-M. L.; Vidal, L.; Dentzer, J.; Gadiou, R.; Märkle, W.; Novák, P.; Spahr, M.; Vix-Guterl, C. Influence of graphite edge crystallographic orientation on the first lithium intercalation in Li-ion battery. Carbon 2015, 91, 458-467.

(28) Pastewka, L.; Malola, S.; Moseler, M.; Koskinen, P. Li + adsorption at prismatic graphite surfaces enhances interlayer cohesion. Journal of Power Sources 2013, 239, $321-325$.

(29) Yildirim, H.; Kinaci, A.; Zhao, Z.-J.; Chan, M. K. Y.; Greeley, J. P. First-Principles Analysis of Defect-Mediated Li Adsorption on Graphene. ACS Applied Materials \& Interfaces 2014, 6, 21141-21150.

(30) Tada, K.; Furuya, S.; Watanabe, K. Ab initio study of hydrogen adsorption to singlewalled carbon nanotubes. Phys. Rev. B 2001, 63, 155405.

(31) Duplock, E. J.; Scheffler, M.; Lindan, P. J. D. Hallmark of Perfect Graphene. Phys. Rev. Lett. 2004, 92, 225502.

(32) Boukhvalov, D. W.; Katsnelson, M. I.; Lichtenstein, A. I. Hydrogen on graphene: Electronic structure, total energy, structural distortions and magnetism from first-principles calculations. Phys. Rev. B 2008, 7r, 035427. 
(33) Peled, E.; Bar Tow, D.; Merson, A.; Gladkich, A.; Burstein, L.; Golodnitsky, D. Composition, depth profiles and lateral distribution of materials in the SEI built on HOPGTOF SIMS and XPS studies. Journal of Power Sources 2001, 97-98, 52 - 57, Proceedings of the 10th International Meeting on Lithium Batteries.

(34) Tsubouchi, S.; Domi, Y.; Doi, T.; Ochida, M.; Nakagawa, H.; Yamanaka, T.; Abe, T.; Ogumi, Z. Spectroscopic Characterization of Surface Films Formed on Edge Plane Graphite in Ethylene Carbonate-Based Electrolytes Containing Film-Forming Additives. Journal of The Electrochemical Society 2012, 159, A1786-A1790.

(35) Mortensen, J. J.; Hansen, L. B.; Jacobsen, K. W. Real-space grid implementation of the projector augmented wave method. Phys. Rev. B 2005, 71, 035109.

(36) Enkovaara, J. et al. Electronic structure calculations with GPAW: a real-space implementation of the projector augmented-wave method. Journal of Physics: Condensed Matter 2010, 22, 253202.

(37) Larsen, A. H. et al. The atomic simulation environment-a Python library for working with atoms. Journal of Physics: Condensed Matter 2017, 29, 273002.

(38) Hammer, B.; Hansen, L. B.; Nørskov, J. K. Improved adsorption energetics within density-functional theory using revised Perdew-Burke-Ernzerhof functionals. Phys. Rev. B 1999, 59, 7413-7421.

(39) Larsen, A. H.; Vanin, M.; Mortensen, J. J.; Thygesen, K. S.; Jacobsen, K. W. Localized atomic basis set in the projector augmented wave method. Phys. Rev. B 2009, 80, 195112.

(40) Trucano, P.; Chen, R. Structure of graphite by neutron diffraction. Nature 1975, 258, $136-137$. 


\section{Graphical TOC Entry}

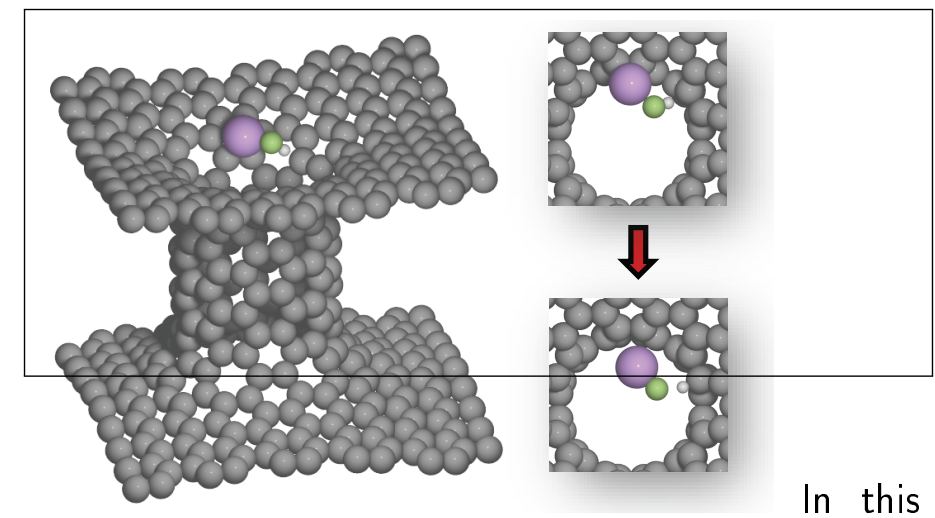

work, we show the effect of the artificial nanostructure of carbon sheets and nanotubes on the reduction reaction of $\mathrm{HF}$ and formation of LiF. 